\title{
ERRATUM TO: RESULTS ON UNIQUENESS OF ENTIRE FUNCTIONS WHOSE CERTAIN DIFFERENCE POLYNOMIALS SHARE A SMALL FUNCTION
}

\author{
P. SAHOO* and H. KARMAKAR \\ Department of Mathematics, University of Kalyani, West Bengal-741235, India \\ e-mails: sahoopulak@yahoo.com, sahoopulak1@gmail.com, himadri394@gmail.com
}

(Received August 3, 2017; accepted November 9, 2017)

Erratum to: Analysis Mathematica 41 (2015), 257-272.

DOI: $10.1007 / \mathrm{s} 10476-015-0302-3$

The following are the corrected statements of the main results of [1].

THEOREM 1. Let $f(z)$ and $g(z)$ be two transcendental entire functions of finite order, and $\alpha(z)(\not \equiv 0)$ be a small function of both $f(z)$ and $g(z)$. Suppose that $\eta$ is a nonzero complex constant, $n(>k), m$ $(\geq 1)$ and $k(\geq 0)$ are integers satisfying $n \geq 2 k+m+6$ when $m \leq k+1$ and $n \geq 4 k-m+10$ when $m>k+1$. If $\left(f^{n}(z)(f(z)-1)^{m} f(z+\eta)\right)^{(k)}$ and $\left(g^{n}(z)(g(z)-1)^{m} g(z+\eta)\right)^{(k)}$ share " $(\alpha(z), 2)$ " and if $f, g$ have no 1-points with multiplicity less than or equal to $k / m$ when $m \leq k$, then either $f(z)=g(z)$ or $f(z)$ and $g(z)$ satisfy the algebraic equation $R(f, g)=0$ where $R(f, g)$ is given by (1.1) of [1].

THEOREM 2. Let $f(z)$ and $g(z)$ be two transcendental entire functions of finite order, and $\alpha(z)(\not \equiv 0)$ be a small function of both $f(z)$ and $g(z)$. Suppose that $\eta$ is a nonzero complex constant, $n(>k), m(\geq 1)$ and $k(\geq 0)$ are integers satisfying $n \geq 3 k+2 m+8$ when $m \leq k+1$ and $n \geq 6 k-m+13$ when $m>k+1$. If $\left(f^{n}(z)(f(z)-1)^{m} f(z+\eta)\right)^{(k)}$ and $\left(g^{n}(z)(g(z)-1)^{m} g(z+\eta)\right)^{(k)}$ share $(\alpha(z), 2)^{*}$ and if $f, g$ have no 1-points with multiplicity less than or equal to $k / m$ when $m \leq k$, then conclusions of Theorem 1 hold.

THEOREM 3. Let $f(z)$ and $g(z)$ be two transcendental entire functions of finite order, and $\alpha(z)(\not \equiv 0)$ be a small function of both $f(z)$ and $g(z)$. Suppose that $\eta$ is a nonzero complex constant, $n(>k), m(\geq 1)$ and $k(\geq 0)$

* Corresponding author. 
are integers satisfying $n \geq 5 k+4 m+12$ if $m \leq k+1$ and $n \geq 10 k-m+19$ if $m>k+1$. If

$$
\begin{aligned}
& \bar{E}_{2)}\left(\alpha(z),\left(f^{n}(z)(f(z)-1)^{m} f(z+\eta)\right)^{(k)}\right) \\
& =\bar{E}_{2)}\left(\alpha(z),\left(g^{n}(z)(g(z)-1)^{m} g(z+\eta)\right)^{(k)}\right)
\end{aligned}
$$

and if $f, g$ have no 1-points with multiplicity less than or equal to $k / m$ when $m \leq k$, then conclusions of Theorem 1 hold.

In the proof of Theorem 1 (see [1, p. 267]), the authors claimed in Case 1 when $B=-1$, after obtaining $F G=1$, that,

$$
\left(f^{n}(z)(f(z)-1)^{m} f(z+\eta)\right)^{(k)}\left(g^{n}(z)(g(z)-1)^{m} g(z+\eta)\right)^{(k)}=\alpha^{2}
$$

gives $N(r, 0 ; f)=S(r, f)$ and $N(r, 1 ; f)=S(r, f)$, which might not be the case always. The argument is thus corrected as follows:

We have

$$
\left(f^{n}(z)(f(z)-1)^{m} f(z+\eta)\right)^{(k)}\left(g^{n}(z)(g(z)-1)^{m} g(z+\eta)\right)^{(k)}=\alpha^{2} .
$$

From the above, we easily see that $N(r, 0 ; f)=S(r, f)$ as $n>k$ and $N(r, 1 ; f)$ $=S(r, f)$ as $f, g$ have no 1-points with multiplicity less than or equal to $\mathrm{k} / \mathrm{m}$ when $m \leq k$.

The extra condition is needed in the other two theorems as their proofs make use of a similar argument as given above.

\section{References}

[1] P. Sahoo and H. Karmakar, Results on uniqueness of entire functions whose certain difference polynomials share a small function, Anal. Math., 41 (2015), 257-272.

The online version of the original article can be found under DOI: $10.1007 / \mathrm{s} 10476-015-0302-3$ 